\title{
Crystal structure of the human thioredoxin reductase-thioredoxin complex
}

\author{
Karin Fritz-Wolf1,2,*, Sebastian Kehr ${ }^{1, \star}$, Michaela Stumpf', Stefan Rahlfs' \& Katja Becker ${ }^{1}$
}

Thioredoxin reductase 1 (TrXR1) is a homodimeric flavoprotein crucially involved in the regulation of cellular redox homeostasis, growth, and differentiation. Its importance in various diseases makes TrxR1 a highly interesting drug target. Here we present the first crystal structures of human TrxR1 in complex with its substrate thioredoxin (Trx). The carboxy-terminal redox centre is found about $20 \AA$ apart from the amino-terminal redox centre, with no major conformational changes in TrxR or Trx. Thus, our structure confirms that the enzyme uses a flexible C-terminal arm for electron transport to its substrates, which is stabilized by a guiding bar for controlled transfer. This notion is supported by mutational analyses. Furthermore, essential residues of the interface region were characterized both structurally and functionally. The structure provides templates for future drug design, and contributes to our understanding of redox regulatory processes in mammals.

\footnotetext{
${ }^{1}$ Interdisciplinary Research Centre, Justus Liebig University, D-35392 Giessen, Germany. ${ }^{2}$ Max Planck Institute for Medical Research, D-69120 Heidelberg, Germany. *These authors contributed equally to this work. Correspondence and requests for materials should be addressed to K.B.

(email: katja.becker@ernaehrung.uni-giessen.de).
} 
T he intracellular redox milieu is supported by the thioredoxin system, composed of the flavoenzyme thioredoxin reductase (TrxR, EC 1.8.1.9), NADPH, and its main substrate thioredoxin (Trx). TrxR reduces the disulphide of Trx using NADPH as the source of reducing equivalents. During catalysis, the electrons are transferred from $\mathrm{NADPH}$ to FAD, then to the buried amino-terminal active site, to the carboxy-terminal active site of the other TrxR subunit, and from there to the substrate at the protein's surface. TrxR influences essential cellular processes either indirectly through reduced thioredoxin or directly by reducing substrates acting in DNA and selenium metabolism, antioxidant defence, or cell growth regulation and apoptosis ${ }^{1}$. The central and diverse functions of the thioredoxin system currently make it one of the most attractive targets for antitumour drug development ${ }^{2,3}$.

In mammals, three types of TrxRs have been characterized: the cytosolic form TrxR1 (also known as TR1 or TXNRD1) ${ }^{4}$, the mitochondrial form TrxR2 (also known as TR3 or TXNRD2) 5 , and thioredoxin glutathione reductase (TGR, also known as TrxR3, TR2 or TXNRD3) that occurs in the microsomal fraction of testis tissue $^{7}$. The hybrid enzyme TGR possesses an N-terminal glutaredoxin-domain in addition to a C-terminal selenocysteine-containing redox centre and therefore combines TrxR and GR (glutathione reductase) activities ${ }^{7}$. Furthermore, mammalian cells contain two ubiquitously expressed $\operatorname{Trxs}{ }^{1,8}$. Trx1 is a cytosolic and nuclear protein, whereas Trx 2 is targeted to mitochondria ${ }^{1,8}$. Cytosolic Trx1 was the major substrate captured by immobilized TrxR1, indicating that the main function of TrxR1 is to reduce $\operatorname{Tr} x 1^{9}$.

TrxRs belong to a family of homodimeric pyridine nucleotide disulphide oxidoreductases with high homology to GRs. The overall fold of human TrxR1 resembles other homodimeric pyridine nucleotide disulphide oxidoreductases such as rat TrxR1 (ref. 10) and human $\mathrm{GR}^{11,12}$. Each subunit contains a binding domain for FAD and $\mathrm{NADPH}$, an interface domain, and a discrete active centre ${ }^{10,13}$. Human TrxR1 and GR share a high active site residue similarity at the cofactor and substrate binding sites as well as a similar reaction mechanism with respect to the reductive half-reaction ${ }^{10}$. The structural and functional characteristics of GR have been studied for many years, and detailed information on the mechanism is available ${ }^{12}$. However, much less is known about the oxidative half-reaction of TrxRs.

Mammalian TrxRs contain selenocysteine (Sec) as the penultimate amino acid, which is important for high catalytic efficiency ${ }^{14}$. The species-specific usage of the selenocysteine insertion sequence element makes a heterologous expression of the mammalian enzyme difficult-a challenge which has been tackled by different groups. Recently, Cheng et al. ${ }^{15}$ solved two crystal structures of selenocysteine containing rat TrxR1 (rTrxR1), one with an oxidized and the other one with a reduced C-terminal active site. The overall structures are similar to other previously published crystal structures of mutated TrxR1, where the selenium-atom is replaced by a sulphur atom $^{10,13,16}$ (Debreczeni JE et al., unpublished result. PDB code 2cfy). In the rTrxR1 structure with the oxidized C-terminal active site, the selenenyl-sulphide motif is found near the side chain of Tyr116 $(\mathrm{PDB} \text { code } 3 \mathrm{eao})^{15}$. This course of the $\mathrm{C}$-terminal arm is similar to the conformation in subunit E of the crystal structure of a U498C mutant of hTrxR1 (PDB code $2 \mathrm{j} 3 \mathrm{n})^{16}$, which also contains the disulphide motif near the side chain of Tyr-116. The similar conformation of the oxidized C-terminal arm in the hTrxR $1^{\mathrm{U} 498 \mathrm{C}}$ mutant as well as in the Sec-containing $r \operatorname{TrxR} 1$ wild-type structure indicate that the hTrxR $1^{\mathrm{U} 498 \mathrm{C}}$ mutant can be used to study mechanistic details.

Using double mutants of hTrxR $1^{\mathrm{C} 497 \mathrm{~S}, \mathrm{U} 498 \mathrm{C}}$ and $\mathrm{hTrx} 1^{\mathrm{C} 35 \mathrm{~S}, \mathrm{C} 73 \mathrm{~S}}$, we trapped the intermediate state of the oxidative half-reaction. We obtained crystals from this mutant protein complex and solved the three-dimensional structure by X-ray analysis at a resolution down to $2.2 \AA$. Our observed complex structure differs fundamentally from all previously published models of the hTrxR1-hTrx 1 complex and provides new insights into the catalytic mechanism of large, eukaryotic TrxRs.

\section{Results}

Generation and purification of the hTrxR1-hTrx1 complex. To catch human TrxR1 in an intermediate state where its substrate thioredoxin is bound, a stabilization of the complex is required. Therefore, we created three C-terminal active site mutants of hTrxR1 (hTrxR1 $1^{\mathrm{C} 497 \mathrm{~S}, \mathrm{U} 498 \mathrm{C}}$, hTrxR1 $1^{\mathrm{U} 498 \mathrm{~S}}$, and hTrxR1 ${ }^{\text {C497_trunc}}$ ) and tested them against three mutants of hTrx $1\left(h \operatorname{Trx} 1^{\mathrm{C} 735}, \mathrm{hTrx} 1^{\mathrm{C} 32 \mathrm{~S}, \mathrm{C} 73 \mathrm{~S}}\right.$, and hTrx1 $1^{\mathrm{C} 35 \mathrm{~S}, \mathrm{C} 73 \mathrm{~S}}$; Methods section). For complex formation we incubated the hTrxR1 mutants with NADPH and the respective hTrx1 mutants. Under the conditions tested, only hTrxR1 C497S,U498C and $\mathrm{hTr} \times 1^{\mathrm{C} 35 \mathrm{~S}, \mathrm{C} 73 \mathrm{~S}}$ were able to form a complex that could be isolated by gel filtration chromatography and was stable in non-reducing SDSPAGE (Fig. 1). This protein sample yielded crystals of the hTrxR1hTrx1 complex that could be analysed by X-ray crystallography.

Structure determination. We obtained monoclinic (space group $\mathrm{P} 2_{1}$ ) and orthorhombic crystals (space group $\mathrm{P} 2_{1} 2_{1} 2_{1}$ ) of hTrxR $1^{\mathrm{C} 497 \mathrm{~S}, \mathrm{U} 498 \mathrm{C}}$ in complex with its substrate hTrx $1^{\mathrm{C} 35 \mathrm{~S}, \mathrm{C} 73 \mathrm{~S}}$ (Table 1). The asymmetric unit of both crystal types contained two copies of the hTrxR1-hTrx1 complex, thereby maintaining the usual TrxR dimer. The monoclinic structure was solved at $2.6 \AA$ resolution via the molecular replacement method, using hTrx 1 (PDB code 1aiu) ${ }^{17}$ and hTrxR1 (PDB code $\left.2 \mathrm{j} 3 \mathrm{n}\right)^{16}$ as search models. Interestingly, all automatic program packages identified the correct position of the hTrxR1 dimer but failed to identify any of the hTrx1 substrates. However, the $2 F o-F c$ map revealed regions of helical density that served to manually place one hTrx1 molecule. The second hTrx1 molecule was inserted into the asymmetric unit through an educated guess (Methods section) so that the two

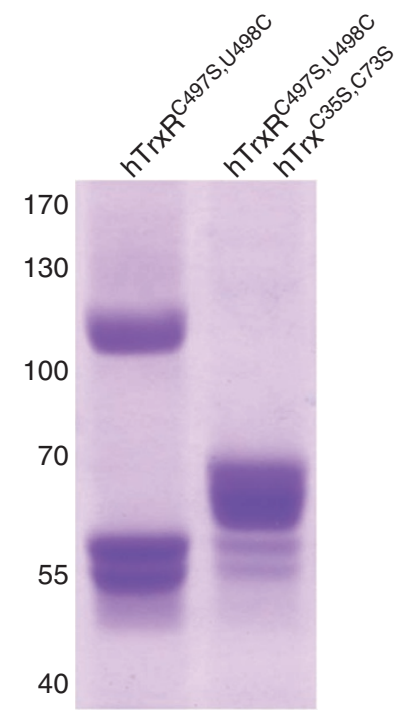

Figure 1 | hTrxR1 ${ }^{\text {C4975,U498c }}$ forms a stable complex with $\mathrm{hTrx} \mathbf{1}^{\mathrm{c35s}, \mathrm{C735}}$. Coomassie staining of a non-reducing 4-12\% SDS-PAGE gel. The left

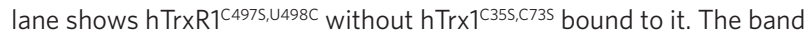
$\sim 114 \mathrm{kDa}$ corresponds to the dimer, the band $\sim 57 \mathrm{kDa}$ to the monomer of

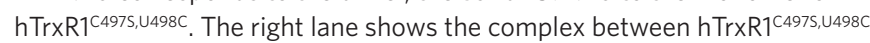

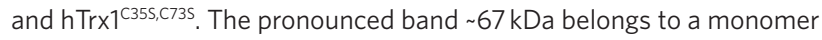
band of $h \operatorname{Tr} x R 1^{C 4975, U 498 C}$ with one molecule of $h \operatorname{Trx} 1^{\mathrm{C35S,C735}}$ bound to it. The lower and weaker bands around $\sim 57 \mathrm{kDa}$ of this non-reducing gel belong

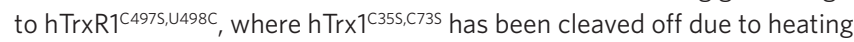
of the sample. The amount of free, uncomplexed $h \operatorname{Tr} \times R 1^{14975, U 498 \mathrm{C}}$ grew with increasing incubation time at $95^{\circ} \mathrm{C}$ before sample loading (data not shown). Conformational changes and changes in interacting forces, especially in the C-terminal region of hTrxR1 upon binding of $h \operatorname{Trx} 1$ are most likely the reason for the absence of a TrxR-homodimer band in this non-reducing SDS gel. 


\begin{tabular}{|c|c|c|}
\hline & \multicolumn{2}{|c|}{ hTrxR1 ${ }^{\mathrm{c} 4975, \mathrm{U} 498 \mathrm{C}}$ complexed with $\operatorname{Trx} 1^{\mathrm{c35s}, \mathrm{C} 73 \mathrm{~S}}$} \\
\hline & Crystal 1 & Crystal 2 \\
\hline \multicolumn{3}{|l|}{ Data collection } \\
\hline Space group & $\mathrm{P} 22_{1} 2_{1} \mathrm{Z}_{1}$ & $\mathrm{P} 2_{1}$ \\
\hline \multicolumn{3}{|l|}{ Cell dimensions } \\
\hline$a, b, c(\AA)$ & $97.25,105.03,120.73$ & $71.3,121.7,73.9$ \\
\hline$\alpha, \beta, \gamma\left(^{\circ}\right)$ & $90,90,90$ & $90,95.3,90$ \\
\hline Resolution $(\AA)$ & $25.0-2.2(2.3-2.2)$ & $50-2.6(2.67-2.60)$ \\
\hline$R_{\mathrm{sym}}(\%)$ & $8.7(29.6)$ & $14.1(65.9)$ \\
\hline$|/ \sigma|$ & $8.3(3.4)$ & $8.3(3.4)$ \\
\hline Completeness (\%) & $84.3(85.3)$ & $99.9(99.9)$ \\
\hline Redundancy & $3.3(3.1)$ & $7.3(7.3)$ \\
\hline \multicolumn{3}{|l|}{ Refinement } \\
\hline Resolution $(\AA)$ & $25.0-2.2$ & $50-2.6$ \\
\hline No. of reflections & 53,469 & 38,626 \\
\hline$R_{\text {work }} / R_{\text {free }}$ & $0.228 / 0.277$ & $0.235 / 0.297$ \\
\hline \multicolumn{3}{|l|}{ No. of atoms } \\
\hline Proteins & 9,254 & 9,238 \\
\hline Ligand/ion & 142 & 142 \\
\hline Water & 425 & 241 \\
\hline Mean $B$-factor $\left(\AA^{2}\right)$ & 40.4 & 52.7 \\
\hline \multicolumn{3}{|l|}{ R.m.s. deviations } \\
\hline Bond lengths $(\AA)$ & 0.01 & 0.01 \\
\hline Bond angles $\left({ }^{\circ}\right)$ & 1.2 & 1.3 \\
\hline
\end{tabular}

complexes in the asymmetric unit were similar. The orthorhombic structure was solved by the molecular replacement method, using the structure of the monoclinic crystals as a search model. The overall temperature factors of the structures were 52.7 (monoclinic) and 40.4 (orthorhombic) $\AA^{2}$. The structures were refined to $\mathrm{R}_{\text {free }}=29.7 \%$ (monoclinic) and $27.7 \%$ (orthorhombic) (Table 1). The monoclinic and orthorhombic structures are essentially similar-with an r.m.s. of $0.6 \AA$ for $1,194 \mathrm{C}_{\alpha}$-atoms of two complexes. Differences were mainly due to crystal packing forces and due to the position of the substrate hTrxl (Fig. 2) and some loops at the surface. Except for some (5-to-8) N-terminally located residues of hTrxR1, the structures were well defined by the electron density. In particular, all cysteine residues were clearly identified with temperature factors below the overall mean.

Overall structure. The homodimeric flavoprotein TrxR1 possesses two redox centres, both of which are required for catalysis. The N-terminally located centre (Cys59 ${ }^{\mathrm{hTrR} 1}$, Cys64 $4^{\mathrm{hTrR} 1}$ ) is buried in the protein, whereas the second, C-terminally located centre (Cys497 ${ }^{\prime h_{T r x R}}$, Sec498 ${ }^{\text {hTrxR1 }}$ ) is positioned on a flexible, highly accessible C-terminal arm of the other subunit (residues belonging to the other subunit are primed). As described above, we used mutants of hTrxR1 (hTrxR1 $\left.1^{\mathrm{C} 497 \mathrm{~S}, \mathrm{U} 498 \mathrm{C}}\right)$ and hTrx1 (hTrx $\left.1^{\mathrm{C} 35 \mathrm{~S}, \mathrm{C} 73 \mathrm{~S}}\right)$ to obtain crystals of the reaction intermediate between hTrxR1 and hTrx1. Both crystal types (monoclinic and orthorhombic) contained two 1:1 hTrxR1-hTrx1 complexes in the asymmetric unit. The two hTrxR1 molecules formed a twofold dimer as previously observed (Fig. 3) $)^{10,13,16}$.

As shown by our structure, complex formation of hTrxR1 with hTrx 1 involves the generation of an intermolecular disulphide bond between the catalytic residues Cys $32^{\mathrm{hTrx}}$ and $\mathrm{U}_{498 \mathrm{C}^{\prime \mathrm{hTrxR}} \text {, which }}$ is supported by their neighbouring residues. The formation of the complex is further stabilized by several interactions between hTrx 1 residues $58^{\mathrm{hTrx} 1}$ to $74^{\mathrm{hTrx} 1}$ and the helical hTrxR1 residues Glu103 ${ }^{\mathrm{hTrxR} 1}$ to Glu122 $2^{\text {hrxR1 }}$ (Fig. 4). Owing to complex formation, Trp $31^{\text {hTrx1 }}$ and

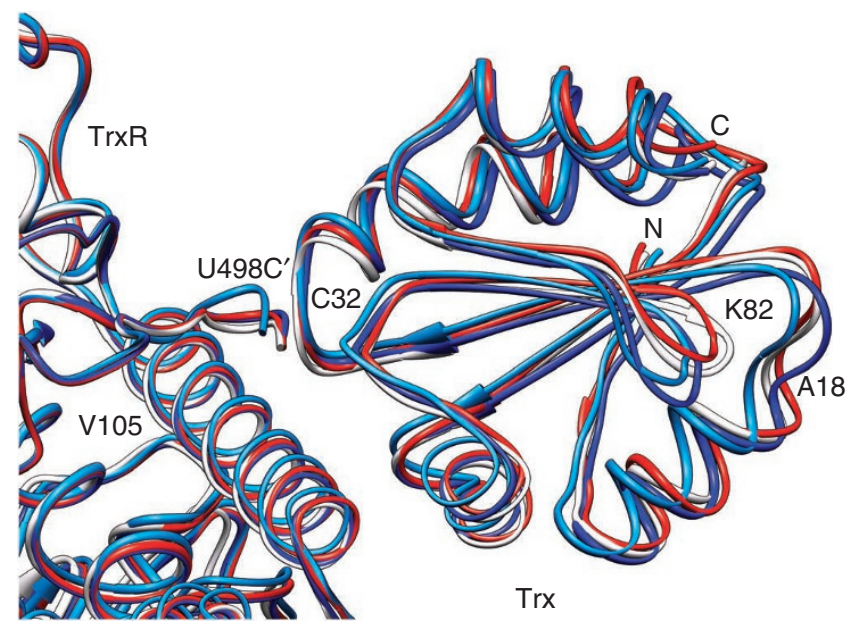

Figure 2 | Superimposed structures of the four hTrxR-hTrx complexes. We used a ribbon representation to show the different positions of the Trx molecules in the four complexes (orthorhombic: grey and cyan; monoclinic: blue and red). There are only slight differences around the intra-molecular disulphide between $\mathrm{U} 498 \mathrm{C}^{\text {'hTrxR1 }}$ and $\mathrm{C} 32^{\mathrm{h} T \times 1}$ but for example in the regions around $\mathrm{K} 82$ and around $\mathrm{A} 18$ the distance between $C \alpha$-atoms increases up to $4 \AA$.

$\operatorname{Trp} 114^{\mathrm{hTrxR} 1}$ are buried with their indole rings at the interface region within van der Waals distance. Trp3 ${ }^{\mathrm{h} T r x 1}$ fits into a pocket, formed by residues Ala29 ${ }^{\text {hrrx1 }}$, Met $74^{\mathrm{hTrx} 1}$, Asn $107^{\mathrm{hTrxR} 1}$, Gly110 ${ }^{\mathrm{hTrxR} 1}$, Ser$111^{\mathrm{hTrxR} 1}$, Trp $114^{\mathrm{hTrxR} 1}$, and the mixed disulphide, whereas the indole is stacked with main chain atoms of residues $110^{\mathrm{hTrxR} 1}$ and $111^{\mathrm{hTrxR} 1}$ (Fig. 5). Moreover, Trp $31^{\mathrm{hTrx} 1}$ forms hydrogen bonds to Asp $60^{\mathrm{hTrx} 1}$, which forms salt bridges to Arg117 hrxR1 in three copies of the four hTrxR1-hTrx1 complexes. The pocket residue $\operatorname{Trp} 114^{\mathrm{hTr} x R 1}$ is sandwiched between Met74 $4^{\mathrm{hTr} 1}$ and Val59 $9^{\mathrm{hTrx}}$ and surrounded by residues $71^{\mathrm{hTrx} 1}, 72^{\mathrm{hTrx} 1}, 117^{\mathrm{hTrxR} 1}$, and $499^{\text {hTrxR1 }}$ (Fig. 4).

Another interaction region in the complex involves several oppositely charged residues: Lys $36^{\mathrm{hTrx}}-$ Glu $103^{\mathrm{hTrxR}}$, Asp $60^{\mathrm{hTrx1}}-$

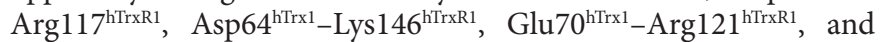
Lys72 $2^{\mathrm{hTrx}}-$ Glu $122^{\mathrm{hTrxR} 1}$. Most of these residues are found in different conformations and interact through van der Waals forces, salt bridges, or hydrogen bonds. A third interaction region involves a set of inter- and intra-molecular, antiparallel $\beta$-bridges formed between hTrxR1 residues $495^{\prime \text { hTrxR1 }}$ to $499^{\prime \text { hTrxR1 }}$ and hTrx1 residues $72^{\text {hTrx1 }}$ to $75^{\text {hTrx1 }}$ (Fig. 6). The active site residue C497S ${ }^{\text {hTrxR1 }}$ is sandwiched between Ser415 $5^{\mathrm{hTrxR} 1}$ and Ser $111^{\mathrm{hTrxR} 1}$, the latter being in hydrogen bond distance ( $3.6 \AA$ ) to His $108^{\text {hTrxR1 }}$ (Fig. 6). The C-terminal region of hTrxR1 is further stabilized by the interaction of residues $494^{\prime \mathrm{hTrxR} 1}$ and $495^{\prime \mathrm{hTrxR} 1}$ with the previously defined 'guiding

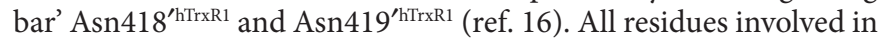
forming the intermediate are highly conserved in mammals.

Structural comparison. The four independent hTrxR1 structures determined in the two crystal forms are essentially identical within the coordinate error $(0.2 \AA$ for $490 \mathrm{C} \alpha$-atoms). Minor variability occurs in the interface region. The first $9 \mathrm{~N}$-terminal residues are not always seen in the electron density and have been omitted from the comparisons. For the substrate hTrx1, a slightly higher structural variation is observed due to the slightly more flexible region $58^{\mathrm{hTrx1}}$ to $74^{\mathrm{hTrx} 1}$ ( $0.6 \AA$ for $105 \mathrm{C} \alpha$-atoms). A comparison of hTrxR1 dimers as seen in our complex structures with published TrxR1 structures ${ }^{10,13,15,16}$ (Debreczeni JE et al., unpublished result. PDB code 2cfy) reveals a typical r.m.s. of $0.5 \AA$. About 965 to 976 equivalent residues were automatically determined. Non-equivalent or non-existing residues were identified near the $\mathrm{N}$ - and C-terminal 


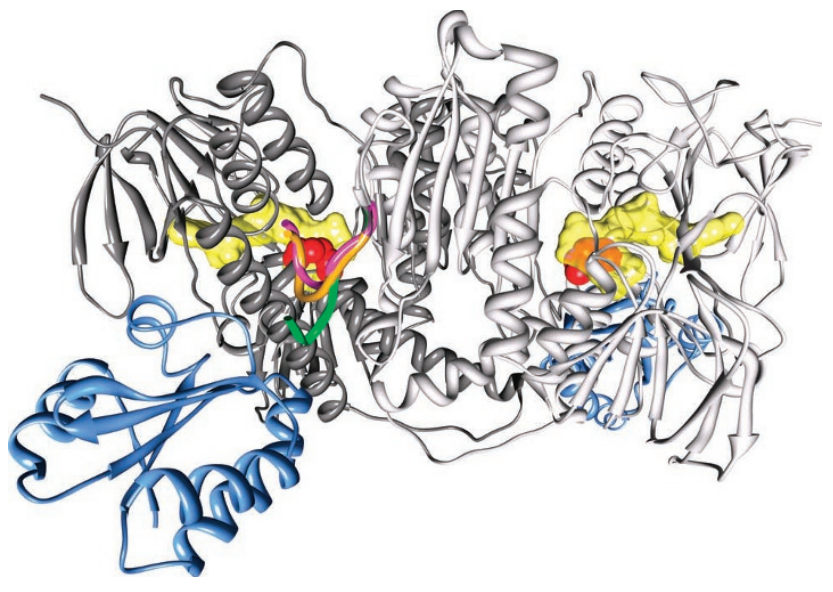

Figure 3 | Overview of the $\mathbf{h}$ TrxR1 dimer with bound $\mathbf{h} \operatorname{Trx} \mathbf{1}$. $h \operatorname{Tr} x \mathrm{R} 1$ (grey) and $h \operatorname{Trx} 1$ (blue) are shown in ribbon representation. FAD (yellow) and the $\mathrm{N}$-terminal redox centre (red) are shown in ball representation. The different conformations of the $\mathrm{C}$-terminus (last 8 residues) as found in the 'oxidized waiting position', 'reduced waiting position', and in the hTrxR1-hTrx1 complex are coloured magenta, orange, and green, respectively. Molecular graphics images were produced using the UCSF Chimera package ${ }^{40}$.

regions. Superimposition of the hTrxl structures obtained in the hTrxR1-hTrx1 complexes with published structures in the uncomplexed form (PDB codes $2 \mathrm{hsh}^{18}$, and $1 \mathrm{aiu}^{17}$ ) also shows r.m.s. deviations of around $0.6 \AA$. A comparison to the hybrid enzyme TGR reveals a slightly higher r.m.s. of $0.9 \AA$ (PDB code $2 \times 8 c)^{19}$.

Apparently, complex formation does not induce major conformational changes in hTrxR1 or hTrx1. Structural changes seen in the complex are confined to the flexible C-terminal arm of hTrxR1. In our previously published structure of hTrxR1, we showed different distinct conformations of the C-terminal $\mathrm{arm}^{16}$. In one conformation, the so-called 'reduced waiting position', Cys497'hTrxR1 and Cys $498^{\prime \mathrm{hTrxR} 1}$ are reduced, but the sulphur atoms are still oriented towards the inter-subunit cleft (Fig. 4; PDB code 2j3n (ref. 16), chain C, residues: $495^{\text {hTrxR1 }}$ to $499^{\text {'hTrRR1 }}$ ). The negative charge at the C-terminus opposes Glu122 $2^{\mathrm{hTrRR}}$, which forms a barrier so that the mobile arm is prevented from leaving the interior of the TrxR molecule before a substrate is bound (Fig. 4). A comparison to this previously published structure of hTrxR1 shows that upon binding oxidized hTrxls, as seen in the complex structure, the side chain of Glu122 ${ }^{\mathrm{hTrxR} 1}$ rearranges. In one copy of our four complexes, Glu122 $2^{\text {hTrRR1 }}$ interacts with Lys72 ${ }^{\text {hrxx1 }}$; in others it points towards the solvent. Upon formation of the complex, the course of the C-terminal arm moves from its 'reduced waiting position' to the surface of hTrxR1. Its motion involves a rotation of residues $494^{\prime \text { hTrxR1 }}$ to $496^{\prime \mathrm{hTrxR}}$, which are still in contact with the guiding bar, and the last three residues $497^{\prime \mathrm{hTrxR} 1}$ to $499^{\prime \mathrm{hTrxR} 1}$ flip to the surface so that after rearrangement residues $495^{\prime \mathrm{hTrxR1}}$ to $499^{\prime \mathrm{hTrxR} 1}$ form a beta turn (Figs 3-5). In this structure, the C-terminal redox centre is found at least $20 \AA$ away from the N-terminal redox centre. In the structure of the 'reduced waiting position' as well as in other structures with a reduced C-terminal active site (TGR, PDB code $2 \times 8 \mathrm{c}$ (ref. 19); rTrxR, PDB code 3ean (ref. 15)) the distance between the redox centres is about $13 \AA$ (Fig. 3). Furthermore, in all of these structures the $\mathrm{C}$-terminal arm is aligned by the conserved guiding bar residue Asn419 ${ }^{\text {hTrRR1 }}$ (Asn518 in TGR) through a strong hydrogen bond.

Functional characterization of hTrxR1 variants. In order to study the impact of the guiding bar residues (Trp407'hTrxR1, Asn $418^{\prime{ }^{\mathrm{hTrxR}} 1}$, and Asn419' ${ }^{\text {hTrxR1 }}$ ) of hTrxR1 upon catalysis, we constructed two different mutants of hTrxR1: hTrxR1 ${ }^{\mathrm{N} 419 \mathrm{~A}, \mathrm{U} 498 \mathrm{C}}$,

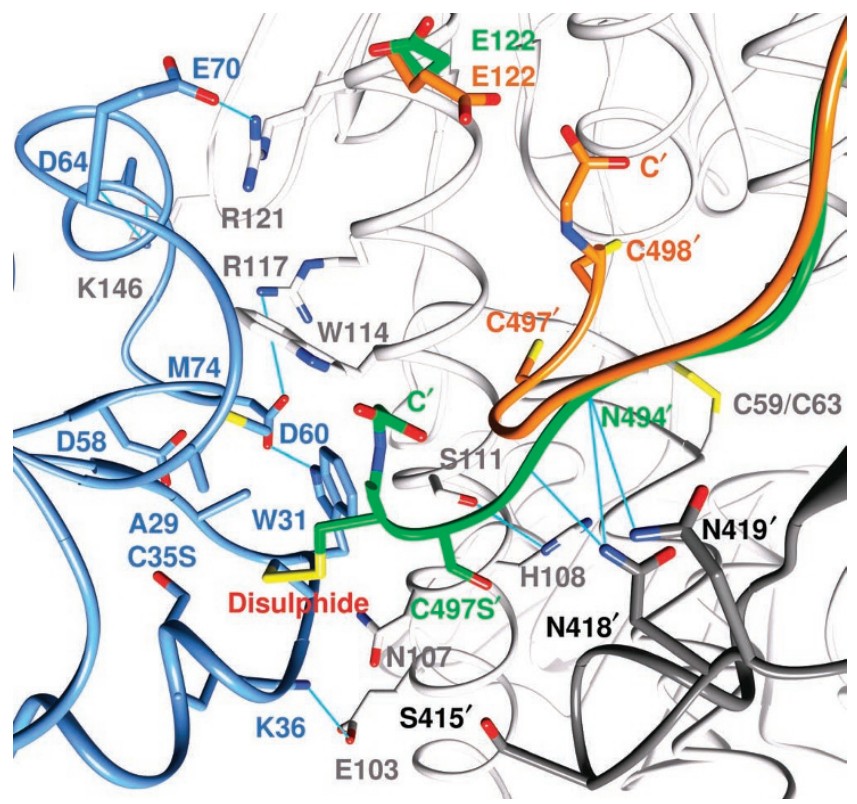

Figure 4 | Close-up view of the hTrxR1-hTrx1 interface. One hTrxR1 subunit is shown in light grey, the other in dark grey and the hTrx1 molecule in blue; the C-terminal arm of the complex structure is shown in green. The conformation of the C-terminal arm and of residue E122 as found in the 'reduced waiting position' are superimposed and are coloured orange. $\mathrm{C}$-termini are labelled with $\mathrm{C}^{\prime}$, the $\mathrm{N}$-terminal redox centre with $\mathrm{C} 59 / \mathrm{C} 63$, and the intra-molecular disulphide between U498C ${ }^{\prime h T \times R 1}$ and C $32^{\text {hTr1 }}$ with disulphide.

and hTrxR1 $1^{\mathrm{W} 407 \mathrm{~A}, \mathrm{~N} 418 / 9 \mathrm{~A}, \mathrm{U} 498 \mathrm{C}}$ (Table 2). Our crystal structures suggest that the guiding bar suppresses random motions of the flexible C-terminal arm of TrxR during catalysis ${ }^{16}$. A disruption of the guiding bar might therefore lead to a higher flexibility of the C-terminal arm. In fact, mutational disruption of the guiding bar did leave to a clear increase in hTrx1- and DTNB-reduction activity of hTrxR1 (Table 2). As expected, the strictly conserved Asn419'hTrxR1 appears to be the most important residue for aligning the flexible $\mathrm{C}$-terminal arm during catalysis, because a simultaneous mutation of all three guiding bar residues (Trp407 $7^{\prime \mathrm{hTrRR}_{1}}$, Asn418 ${ }^{\prime \mathrm{hTrxR} 1}$, and Asn419 ${ }^{\prime \mathrm{hTrxR} 1}$ ) led only to a minor increase in hTrx1- and DTNB-reduction activity of hTrxR1 compared to the mutation of Asn419 ${ }^{\text {hTrxR1 }}$ alone (Table 2).

The interface residue Trp $114^{\mathrm{hTrxR} 1}$ is solvent exposed in uncomplexed hTrxR1 structures, but it is interlocked with several Trxresidues in the complex structure (Figs 4 and 6). In order to investigate the importance of $\operatorname{Trp} 114^{\mathrm{hTrR} 1}$ for Trx substrate binding, we mutated Trp114 to Ala. As expected, hTrxR1 ${ }^{\text {W114A,U498C }}$ did not have detectable activity with hTrx $1^{\mathrm{C} 73 \mathrm{~S}}$ (Table 2). Furthermore, we determined increased NADPH-oxidation with high concentrations of hTrxR $1^{\mathrm{W} 114 \mathrm{~A}, \mathrm{U} 498 \mathrm{C}}$ in the presence and absence of $\mathrm{hTrx} 1^{\mathrm{C} 73 \mathrm{~S}}$ in the assay (Table 2). Our results are in accordance with the previously described findings that TrxR1 shows an enhanced NADPH oxidation if there is no electron transport to the substrate $e^{20,21}$. Our notion was further supported by the fact that with DTNB, a small artificial disulphide substrate ${ }^{22}$, hTrxR $1^{\mathrm{W} 114 \mathrm{~A}, \mathrm{U} 498 \mathrm{C}}$ showed the same kinetic constants as $\mathrm{hTrxR} 1^{\mathrm{U} 498 \mathrm{C}}$, because $\operatorname{Trp} 114^{\mathrm{hTrRR} 1}$ is not involved in binding of DTNB to the C-terminal active site (Table 2).

\section{Discussion}

This is the first time the complex of a large, eukaryotic thioredoxin reductase, with its physiological substrate thioredoxin, has been described. We used mutants of hTrxR1 (hTrxR1 ${ }^{\mathrm{C} 497 \mathrm{~S}, \mathrm{U} 498 \mathrm{C}}$ ) and hTrx1 $\left(\mathrm{hTrx} 1^{\mathrm{C} 35 \mathrm{~S}, \mathrm{C} 73 \mathrm{~S}}\right)$ to obtain a stable hTrxR1-hTrx1 complex. The crys- 

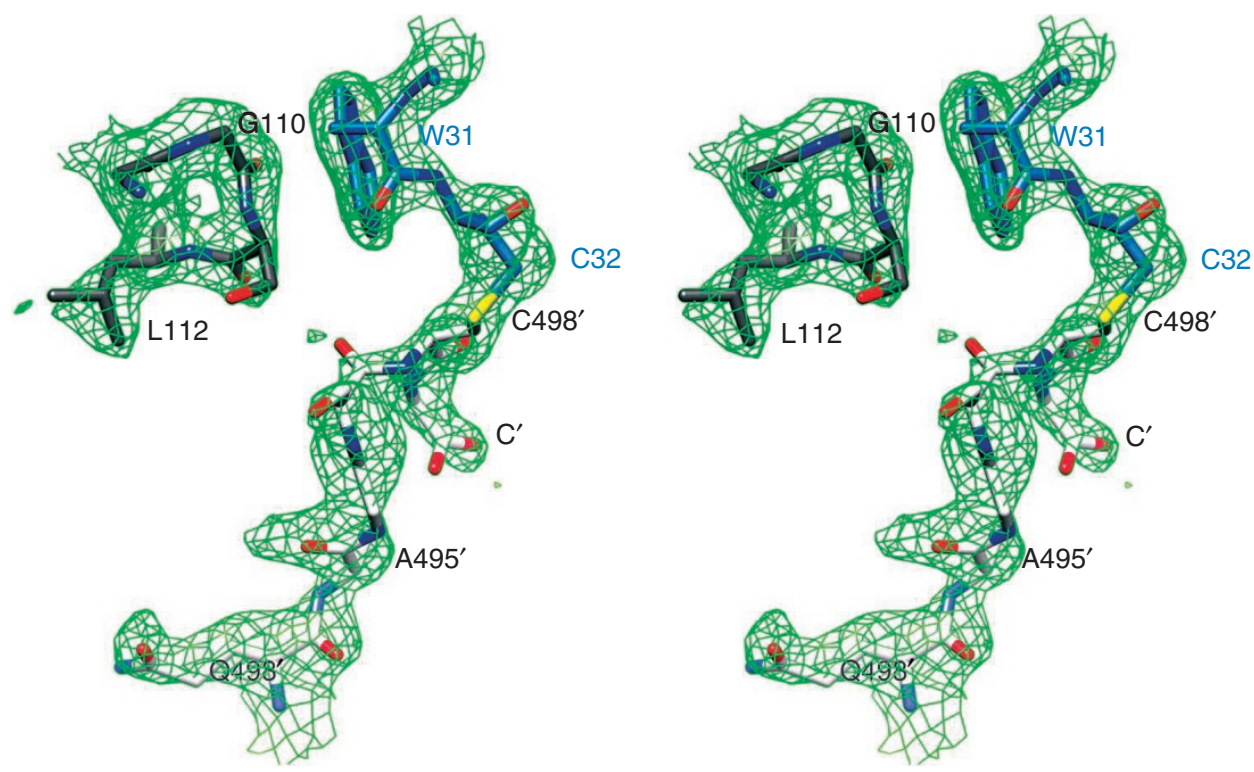

Figure 5 | Stereo view of a composite SA omit map. A composite SA omit map at $2.2 \AA$ resolution and contoured at $1 \sigma$, covering a major part of the interface region of the hTrxR1-hTrx1 complex (hTrxR1, residues labelled in black; hTrx1, residues labelled in blue).

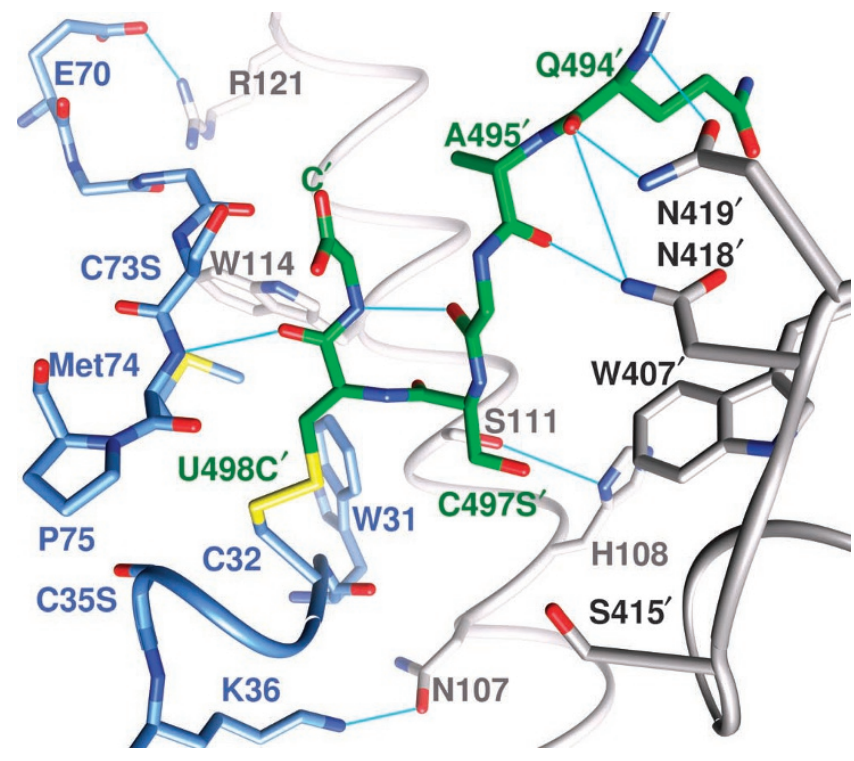

Figure 6 | Close-up view of the intermolecular disulphide bond. Interand intra-molecular, antiparallel $\beta$-bridges between $h \operatorname{Tr} x \mathrm{R} 1$ residues (grey, green) and $\mathrm{h} T r \times 1$ residues (blue) and the mixed disulphide between U498C'hTrRl and Cys32 ${ }^{\text {hTrx }}$ are shown.

tal structures of the complex provide new insight into the electron transport from NADPH to the oxidizing substrate mediated by the enzyme. On the basis of our data, the structures mimic the intermediate state of the mixed disulphide between $\operatorname{TrxR}$ and oxidized $\operatorname{Tr} x$ as delineated below.

In our structures of the hTrxR1-hTrxl complex, the last six C-terminal residues of hTrxR1, including the second redox centre, adopt a well-defined position, and the last four residues are located at the surface of the enzyme (Fig. 3). The C-terminal residues form a set of inter- and intra-molecular, antiparallel $\beta$-bridges between hTrxR1 residues $495^{\text {hTrxR1 }}$ to $499^{\text {hTrxR1 }}$ and hTrx1 residues $72^{\text {hTrx1 }}$ to $75^{\text {hTrx1 }}$ as well as a mixed disulphide between U498C $\mathrm{C}^{\text {hTrxR1 }}$ and Cys32 $2^{\text {hTrx1 }}$ (Fig. 6). It has been postulated before that either residue Sec $498^{\prime \text { hTrxR1 }}$ or residue Cys497 ${ }^{\text {hTrxR1 }}$ attacks the intra-molecular hTrx1-disulphide
(Cys32 ${ }^{\mathrm{hTrx} 1}$, Cys35 $5^{\mathrm{hTrx} 1}$ ), forming an inter-molecular disulphide bond between hTrx1 and hTrxR1 during catalysis ${ }^{23}$. After deprotonation of the other, non-attacking Sec/Cys residue the mixed disulphide is resolved, resulting in the release of reduced hTrx1. The presence of the mixed disulphide observed in all of our crystallographically independent copies of the complex supports the notion that Sec $498^{\prime h T r x R 1}$ is the attacking residue of the intra-molecular disulphide in oxidized hTrx1. Consequently Cys $497^{\prime h T r x R 1}$ must cleave the intermolecular disulphide. Moreover, only the mutant combinations hTrxR $1^{\mathrm{C} 497 \mathrm{~S}, \mathrm{U} 498 \mathrm{C}}$ and $\mathrm{hTrx} 1^{\mathrm{C} 35 \mathrm{~S}, \mathrm{C} 73 \mathrm{~S}}$ yielded a stable complex, indicating that C $32^{\mathrm{hTrx} 1}$ is initially attacked by Sec $498^{\text {'hTrxR1 }}$ of the enzyme.

Interestingly, there is no His/Glu dyad in the vicinity that could be used for the deprotonation essentially involved in resolving a disulphide bond. Instead, Cys497 ${ }^{\text {hTrxR1 }}$ is sandwiched between Ser $415^{\text {hTrxR1 }}$ and Ser111 $11^{\mathrm{hTxR} 1}$, the latter being within hydrogen bond distance $(3.6 \AA)$ to a strictly conserved His $108^{\text {hTrxR1 }}$ (Fig. 6). This structural environment suggests that Cys497/hTrxR1 could be deprotonated by a Ser111/His108 $8^{\text {hTrR1 }}$ dyad. To test this hypothesis, we generated four different hTrxR1 mutants: hTrxR1 ${ }^{\mathrm{H} 108 \mathrm{~L}, \mathrm{U} 498 \mathrm{C}}$, hTrxR1 $^{\text {S111A,U498C }}$, hTrxR $1^{\text {S415A,U498C }}$, and hTrxR1 ${ }^{\text {H108L,S111A,U498C }}$. Surprisingly, all mutants showed $>90 \%$ thioredoxin reducing activity in direct comparison to hTrxR $1^{\mathrm{U} 498 \mathrm{C}}$ (data not shown). This renders the hypothesis of a Ser111/His108 ${ }^{\text {hTrxR1 }}$ dyad for deprotonation of Cys497'hTrxR1 unlikely. Cheng et al. ${ }^{15}$ proposed that deprotonation of Cys497'TrxR occurs either through direct proton transfer from Cys $497^{\prime \operatorname{TrxR}}$ to Cys35 $5^{\text {Trx }}$ or via solvent water molecules. In our structure Cys $497^{\prime \mathrm{hTrRR}}$ and Cys $35^{\mathrm{hTrx} 1}$ are $10 \AA$ apart; this distance is incompatible with a direct proton transfer between these cysteines. Our results, therefore, strongly support the hypothesis that the proton transfer from Cys $497^{\text {TrxR }}$ to Cys $35^{\text {Trx }}$ occurs via solvent water molecules.

The C-terminal redox centre of hTrxR1, located on a flexible arm, has been expected to act as an electron shuttle between the buried N-terminal dithiol and the substrate ${ }^{16,24}$. In our structure of the hTrxR1-hTrx1 complex, the buried dithiol is about $20 \AA$ apart from the $\mathrm{C}$-terminal active site. In uncomplexed structures of human (monE, monC, PDB code $2 \mathrm{j} 3 \mathrm{n})^{16}$ and rat TrxR1 (PDB code 3eao and 3 ean $)^{15}$, this distance decreases to about $13 \AA$, implying that the flexible C-terminal arm adopts different conformations upon catalysis (Figs 3 and 4). One of these conformations could be described as an 'oxidized waiting position' with an oxidized 
Table 2 | Steady-state kinetic constants of different hTrxR1 ${ }^{\mathrm{Uagsc}}$ variants.

\begin{tabular}{|c|c|c|c|c|}
\hline TrxR variants ${ }^{\star}$ & U498C & N419A & W407A, N418/9A & W114A \\
\hline \multicolumn{5}{|l|}{$h \operatorname{Tr} x^{C 735, \uparrow}$} \\
\hline$V_{\max }\left(m U m g^{-1}\right)$ & $57.8 \pm 15.5$ & $155 \pm 24$ & $191 \pm 12$ & $1.6 \pm 0.3 \ddagger$ \\
\hline$k_{\text {cat }}\left(\min ^{-1}\right)$ & $3.2 \pm 0.8$ & $8.5 \pm 1.3$ & $10.5 \pm 0.68$ & $0.09 \pm 0.02 \ddagger$ \\
\hline$K_{m}(\mu \mathrm{M})$ & $11.1 \pm 1.5$ & $21.4 \pm 2.0$ & $29.2 \pm 2.9$ & NAS \\
\hline$k_{\text {cat }} / K_{m}\left(\min ^{-1} \mu M^{-1}\right)$ & $0.24 \pm 0.03$ & $0.37 \pm 0.04$ & $0.35 \pm 0.03$ & NAS \\
\hline \multicolumn{5}{|l|}{ DTNB" } \\
\hline$V_{\max }\left(m U m g^{-1}\right)$ & $357 \pm 50$ & $546 \pm 28$ & $661 \pm 30$ & $439 \pm 14$ \\
\hline $\mathrm{k}_{\text {cat }}\left(\min ^{-1}\right)$ & $19.5 \pm 2.7$ & $29.6 \pm 1.8$ & $36.2 \pm 1.7$ & $23.8 \pm 0.7$ \\
\hline$K_{m}(\mu M)$ & $1,072 \pm 87$ & $1,190 \pm 99$ & $1,155 \pm 60$ & $1,090 \pm 67$ \\
\hline $\mathrm{k}_{\mathrm{cat}} / \mathrm{K}_{\mathrm{m}}\left(\min ^{-1} \mu \mathrm{M}^{-1}\right)$ & $0.018 \pm 0.005$ & $0.025 \pm 0.003$ & $0.031 \pm 0.002$ & $0.022 \pm 0.001$ \\
\hline
\end{tabular}

All values represent means of at least three independent experiments, with each experiment representing at least three independent measurements. The standard deviation (s.d.) is indicated by \pm .

$\star$ All hTrxR mutants bore the U498C mutation. The one with only the U498C mutation was considered the reference protein.

TKinetic parameters were determined following NADPH oxidation spectrophotometrically at $340 \mathrm{~nm}$ in TrxR1-catalysed, NADPH-dependent Trx reduction.

Intrinsic NADPH-oxidase activity.

SNA, not applicable. Given the very low turnover of this variant (NADPH-oxidase activity, see main text for further information), the other parameters cannot be reliably determined.

||Kinetic parameters were determined following TNB- formation spectrophotometrically at $412 \mathrm{~nm}$ in TrxR1-catalysed, NADPH-dependent DTNB reduction.

C-terminal active site (monE, PDB code $2 \mathrm{j} 3 \mathrm{n}^{16}, 3 \mathrm{eao}^{15}$ ) $13 \AA$ apart from the buried disulphide. Upon reduction of the $\mathrm{N}$-terminal active site by NADPH, the C-terminal active site probably turns to the $\mathrm{N}$-terminal dithiol and becomes reduced. We assume that after binding the next NADPH molecule, the re-reduced N-terminal active site repels the reduced C-terminal active site. This confirmation could be described as 'reduced waiting position' (also $13 \AA$ apart from the N-terminal active site) with both side-chain sulphur atoms directed toward the inter-subunit cleft, making an electron transport to the substrate impossible (monC, PDB code $2 \mathrm{j} 3 \mathrm{n})^{16}$. Upon binding the substrate Trx, the C-terminal arm most likely turns to the surface and reduces $\operatorname{Trx}(20 \AA$ apart, Figs 3 and 4). We assume that the oxidized C-terminal redox centre swings back to the conformation seen in the uncomplexed structures of TrxR1, thereby adopting the mentioned 'oxidized waiting position' again, completing the possible reaction cycle. In all conformations, the Cterminal arm forms variable hydrogen bonds to the guiding bar residues (Trp407 $7^{\text {hTrxR1 }}$, Asn418 ${ }^{\text {hTrxR1 }}$, Asn419 ${ }^{\text {hTrxR1 }}$ ). Furthermore, the hydrogen bond, which involves the conserved residue Asn419'hTrxR1, is also present in structures of rat TrxR and TGR with a reduced C-terminal end.

Our hypothesis is strongly supported by kinetic evidence, which we gained by disrupting the residues of the guiding bar (Table 2). Correspondingly, disruption of the guiding bar led to $55 \%$ and $72 \%$ increases in the catalytic efficiency of hTrxR1 for hTrx 1 and for DTNB, respectively (Table 2). Mutation of the strictly conserved guiding bar residue Asn $419^{\prime \mathrm{hTrxR1}}$ alone led to a strong effect (Table 2), rendering it the most important residue for aligning the flexible $\mathrm{C}$ terminal arm during catalysis. Simultaneous mutation of all three

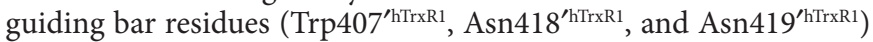
only led to a minor increase in catalytic efficiency of hTrxR1 compared to the Asn419 ${ }^{\text {hTrxR1 }}$ mutation (Table 2). The increase in catalytic efficiency can be explained by the fact that the flexible C-terminal arm is aligned by the guiding bar residues. If these contacts are disrupted through mutations in these residues, the random motions of the C-terminal arm should increase. Consequently, this leads to a faster reduction of its substrates but may also lead to an unspecific loss of electrons.

Our view of the reaction cycle is also in accordance with a number of other biochemical and structural observations. It has been noted that Trxs as well as TrxRs contain solvent-exposed tryptophan residues, which are highly unfavourable energetically. Owing to complex formation, solvent-exposed Trp31 ${ }^{\mathrm{hTrx} 1}$ and $\operatorname{Trp} 114^{\mathrm{hTrxR} 1}$ fit into hydrophobic pockets in the interface region; additionally, their indole rings are in van der Waals contact. The importance of Trp $114^{\mathrm{hT} r x R 1}$ for hTrx1 binding could be supported through kinetic analysis of a hTrxR $1^{\mathrm{W} 114 \mathrm{~A}, \mathrm{U} 498 \mathrm{C}}$ mutant, which did not produce a detectable activity in the Trx-assay (Table 2). Trp $114^{\mathrm{hTrxR} 1}$ interacts with Trp31 $1^{\mathrm{hTrx} 1}$ and other Trx-residues, but it is not involved in binding the small, artificial disulphide substrate DTNB ${ }^{22}$. Hence, if we used DTNB as substrate, the hTrxR1 $1^{\mathrm{W} 114 \mathrm{~A}, \mathrm{U} 498 \mathrm{C}}$ mutation did not influence steady-state kinetic constants compared to the hTrxR $1^{\mathrm{U} 498 \mathrm{C}}$ variant (Table 2). To conclude, we could provide strong evidence that Trp $114^{\text {hTrxR1 }}$ is involved in hTrx1 binding, but not in the catalytic reduction mechanism itself.

In previous reports, Asp $60^{\mathrm{hTrx} 1}$ has been mentioned as an important residue that quenches $\operatorname{Trp} 31^{\mathrm{hTrx} 1}$ (refs 17,25). In our complex structure, Asp $60^{\mathrm{hTrx} 1}$ is further stabilized by forming a salt bridge to Arg117 ${ }^{\text {hTrRR }}$ (Fig. 4) underlining the role of these residues for Trx binding and catalysis. In this work we used a C73S $\mathrm{S}^{\mathrm{hTrx}}$ mutant in order to avoid formation of hTrx1 dimers involving a disulphide between Cys $73^{\mathrm{hTrx} 1}$ and C73 ${ }^{\text {hTrx1 }}$ of a second hTrx1 molecule ${ }^{26,27}$. Such Trx1-dimers are known not to be a substrate of TrxR1 (ref. 26). This finding can be explained by our structure, because in the presence of

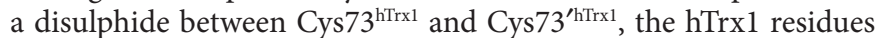
$73^{\text {hTrx } 1}$ to $75^{\text {hTrx1 }}$, which are involved in binding of hTrx1 to hTrxR1 (see above), are not available for hTrxR1-hTrx1 complex formation (Fig. 6). Moreover our structure is likely to explain the considerable drop in hTrx1 activities upon disulphide formation between residues Cys62 $2^{\text {hTrx1 }}$ and Cys69 ${ }^{\text {hTrx }}$ (ref. 28). Both cysteines belong to the interface region $58^{\mathrm{hTrx} 1}$ to $74^{\mathrm{hTrx} 1}$ and are located on the same helix separated by two turns; therefore, disulphide formation distorts the alignment of hTrx1 with hTrxR1. Other residues of the interface region in our structure have also been reported to be of importance. Pro $75^{\text {hTrx } 1}$ has been shown to prevent metal binding by the reactive thiolate-based active site $^{29}$, and residues Glu70 ${ }^{\mathrm{hTrx} 1}$ and Lys $72^{\mathrm{hTrx} 1}$ are required for procaspase- 3 affinity ${ }^{30}$. Lys $36^{\text {hTrx1 }}$ faces Glu103 ${ }^{\text {hTrxR1 }}$ and is probably also involved in the docking and release of hTrx 1 (ref. 31) (Fig. 4). One region in the complex interface involves several oppositely charged residues, indicating their importance for docking and releasing hTrx1. All of the residues involved in forming the complex are strictly conserved in mammals, subsequently underlining their essential role in catalysis.

The broad functional spectrum of TrxR1, its involvement in a magnitude of cellular processes, and therefore, its importance in a variety of diseases make it a highly interesting candidate for drug development ${ }^{7}$. To simultaneously decrease DNA synthesis and antioxidant defence of a tumour cell by the inactivation of one enzyme is indeed a tempting approach. A number of clinically and experimentally utilized chemotherapeutic drugs, including the cytostatic agent BCNU, gold and platinum complexes, and compounds acting at the dimer-interface have already been shown to inhibit TrxR1 effectively ${ }^{2,3,7}$. Chemotherapeutic treatment can be successfully realized by redox targeting, that is, inhibition of $\operatorname{TrxR}$ or $\operatorname{Trx}$, in different forms of human cancer 
such as glioblastoma, colon, and lung and breast cancer. Moreover, it has been shown that $\operatorname{Trx}$ and $\operatorname{TrxR}$ have major roles in the denitrosylation processes controlling apoptosis ${ }^{32}$. Here we have described the structural characteristics of hTrxR1-hTrx 1 complex formation. Complex formation does not induce gross conformational changes in TrxR or Trx. Instead, the enzyme uses a flexible C-terminal arm, which adopts different conformations during catalysis to reduce its substrates. The function of the guiding bar, as postulated in our previous report ${ }^{16}$, could be strengthened by the crystal structure, and disruption of the guiding bar led to an increase in Trx-reduction efficiency, which further supports its importance for regulatory processes in catalysis of TrxR1. We believe that the three-dimensional structure of the hTrxR1-hTrx1 complex will facilitate future drug discovery approaches and will significantly contribute to our understanding of redox regulatory processes in mammals.

\section{Methods}

Cloning and purification. Three hTrxR1 constructs were cloned into the pET28a (+) expression vector (Novagen) using NdeI and HindIII restriction sites: a double hTrxR1 mutant where Cys497 and Sec498 were replaced by Ser and Cys, respectively (hTrxR $1^{\mathrm{C} 497 \mathrm{~S}, \mathrm{U} 498 \mathrm{C}}$ ), as well as a hTrxR1 mutant where Sec 498 was replaced by $\operatorname{Ser}\left(\mathrm{hTrxR} 1^{\mathrm{U} 4985}\right)$, and a truncated form of $\mathrm{hTrxR} 1$ where a stop codon

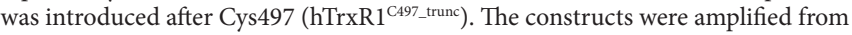
the previously described hTrxR1 ${ }^{\mathrm{U} 498 \mathrm{C}}$ construct, using OhTrxRf $\left(5^{\prime}\right.$-TATTACATA TGAACGGCCCTGAAGATCTTCCCAAGTCC-3') as forward primer, and OhTrxRrSC (5'-TATTAAAGCTTTTAACCACAGGAGCCAGCCTGGAGGAT-3'), OhTrxRrCS (5'-TATTAAAGCTTTTAACCAGAGCAGCCAGCCTGGAGGAT-3'), or OhTrxRrCtrunc (5'-TATTAAAGCTTTTAACCTTAGCAGCCAGCCTGGAG GAT- $3^{\prime}$ ) as reverse primers for PCR, respectively ${ }^{33}$. In the two full-length constructs Sec was mutated either to Cys or Ser to enable high-yield heterologous overexpression in E. coli. The other mutation (C497S) and the truncation as well as the mentioned mutation of $\mathrm{U} 498 \mathrm{~S}$ were introduced to obtain stable protein complexes for crystallization. Recombinant, His-tagged hTrxR1 mutant proteins were overexpressed in E. coli using the pET28a(+)/BL21 Rosetta (DE3) pLysS expression system and purified by Ni-NTA affinity chromatography as described before ${ }^{33}$.

Additionally, three hTrx 1 mutants were used: $h \operatorname{Trx} 1^{\mathrm{C} 733}, \mathrm{hTrx} 1^{\mathrm{C} 325, \mathrm{C} 73 \mathrm{~S}}$, and

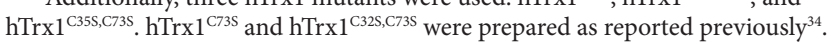
Mutation of Cys 35 to Ser was introduced into $\operatorname{Trx}^{\mathrm{C}}{ }^{\mathrm{C} 3 \mathrm{~S}}$ by PCR with $P f u$ polymerase (Promega) using mutational primers (hTrxC35Sfor: $5^{\prime}$-CGTGGTGTGGGCCT TCCAAAATGATCAAGC-3'; hTrxC35Srev: 5'-GCTTGATCATTTTGGAAG GCCCACACCACG-3'). The recombinant proteins were overexpressed using the pQE30/E. coli M15 expression system and purified by Ni-NTA affinity chromatography. The $\mathrm{C} 73 \mathrm{~S}$ mutation was present in all hTrx1 species to prevent undesirable dimer formation ${ }^{26,27}$.

Complex formation and gel filtration chromatography. hTrxR1 mutant proteins were incubated with a 20-molar excess of hTrx 1 mutant proteins in Tris buffer ( $50 \mathrm{mM}$ Tris- $\mathrm{HCl}, 200 \mathrm{mM} \mathrm{NaCl}, 1 \mathrm{mM}$ EDTA, $\mathrm{pH}$ 7.6) containing $2.5 \mathrm{mM}$ $\mathrm{NADPH}$ for $5 \mathrm{~h}$ at room temperature. After incubation, the protein solution was analysed by gel filtration chromatography on a HiLoad 16/60 Superdex 200 prep grade column (GE Healthcare), which was connected to an ÄKTA-FPLC system (GE Healthcare). FPLC fractions were detected spectrophotometrically, and peak areas and $k_{\mathrm{AV}}$-values were evaluated using the software UNICORN 4.11 (GE Healthcare). Protein complex containing FPLC fractions were analysed by nonreducing 10\% SDS-polyacrylamide gel electrophoresis (SDS-PAGE).

Crystallization. Mutants (see above) of hTrxR1, complexed with hTrx1 in $50 \mathrm{mM}$ Tris- $\mathrm{HCl}, 200 \mathrm{mM} \mathrm{NaCl}, 1 \mathrm{mM}$ EDTA, $\mathrm{pH}$ 7.6, were concentrated to $10 \mathrm{mg} \mathrm{ml}^{-1}$. Crystals were grown at $24^{\circ} \mathrm{C}$ in hanging drops by the vapour diffusion technique. The reservoir of the monoclinic crystals contained 15\% PEG 4000, and the reservoir of orthorhombic crystals contained 15\% PEG 6000, MES 0.1 M, pH 6.5. Before the measurements, the crystals were soaked in mother liquour containing a final concentration of $20 \%$ glycerol.

Data collection. All diffraction data were recorded by the rotation method and processed with $\mathrm{XDS}^{35}$. We obtained monoclinic (space group $\mathrm{P} 2_{1}$ ) and orthorhombic crystals (space group $\mathrm{P}_{1} 2_{1} 2_{1}$ ) of hTrxR1 ${ }^{\mathrm{C} 497, \mathrm{U} 498 \mathrm{C}}$ in complex with its substrate $\mathrm{hTrx} 1^{\mathrm{C} 355, \mathrm{C} 73 \mathrm{~S}}$. The crystals of both types contained two copies of the hTrxR1-hTrx1 complex in the asymmetric unit thereby maintaining the usual hTrxR1 dimer. A complete monoclinic dataset to $2.6 \AA$ resolution and, owing to radiation damage, a less complete orthorhombic dataset to $2.2 \AA$ resolution (Table 1) were collected at $100^{\circ} \mathrm{K}$ using synchrotron radiation (SLS, Paul Scherrer Institute, Villigen, Suisse, beamline X10SA; rotation/image: $1.0^{\circ}, \lambda=1 \AA$ (monoclinic); rotation/image: $1.0^{\circ}$, $\lambda=1.0068 \AA$ (orthorhombic), detector CCD, (MARCCD)).
Structure determination. Reflection phasing and structure refinements were carried out using the cns program suite ${ }^{36}$. Structure solution was started with the monoclinic crystal data (which were available first) using the molecular replacement method. The starting model consisted of the human TrxR1 dimer (PDB code $2 \mathrm{j} 3 \mathrm{n}){ }^{16}$ without the $10 \mathrm{~N}$-terminal and $20 \mathrm{C}$-terminal TrxR residues known to be flexible. The automatic program packages ${ }^{36,37}$ identified the correct positions of the hTrxR1 dimer. The search model was augmented subsequently by the small hTrxl (PDB code 1aiu) ${ }^{17}$ molecules but the programs failed to reveal their correct position, presumably because of the intricate packing of the molecules in the crystal. However, the $2 F o-F c$ map showed regions of helical density that served as an anchor point to position manually one hTrx1 molecule using the interactive graphics program $\mathrm{O}$ (ref. 38). The second $\mathrm{hTrx} 1 \mathrm{molecule}$ was generated by application of the twofold rotation of the TrxR dimer, and subsequent rigid-body refinement. This model (without the last C-terminal residues) was used as a starting model for the orthorhombic crystal form. Refinement was completed after several rounds of model correction, thereby imposing non-crystallographic restraints on residues 15:50, 54:81, 100:106, 135:220, 227:240, 279:335, 342:410, 412:428, and 434:490. Structure assignment of the C-terminal loop was initially based on the best interpretable density region of the two complexes of the orthorhombic crystal forms. After refinement, annealed omit maps were used to correct the course of the C-terminal chain residues in both orthorhombic and monoclinic crystal forms (Fig. 5). The models show good stereo-chemistry (Table 1 ) as verified by procheck ${ }^{39}$. Coordinates and measured reflection amplitudes were deposited in the Brookhaven Protein Data Bank (PDB code 3QFA, and 3QFB).

Construction of further hTrxR1 variants. On the basis of the information provided by the crystal structure of the complex, 3 hTrxR1 mutants were produced from the hTrxR1 $1^{\mathrm{U} 498 \mathrm{C}}$ mutant described above (hTrxR1 ${ }^{\text {W114A,U498C }}, \mathrm{hTrxR}^{\mathrm{N} 419 \mathrm{~A}, \mathrm{U} 498 \mathrm{C}}$, and hTrxR1 $\left.{ }^{\text {W407A,N418/9A,U498C }}\right)$. Mutations were inserted through site-directed mutagenesis using the Quick Change II Site Directed Mutagenesis Kit (Stratagene). Following primers were used to generate the W114A mutation: forward 5'-GCTCTTTGAA TTCGGGCTACCGAGTAG- $3^{\prime}$ and reverse $5^{\prime}$-CTACTCGGTAGCCCGAATTCA

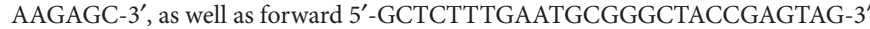
and reverse $5^{\prime}$-CTACTCGGTAGCCCGCATTCAAAGAGC- $3^{\prime}$. Following primers were used to generate the N419A mutation: forward $5^{\prime}$-CCGTCAAGAGATAA CGCCAAATGTTATGCAA- ${ }^{\prime}$ and reverse $5^{\prime}$-TTGCATAACATTTGGCGTTA TCTCTTGACGG- $3^{\prime}$. Following primers were used to generate the N418/9A mutation: forward 5'-GAATGGACGATTCCGTCAAGAGATGCCGCCAAATGTTAT GCAA-3' and reverse 5' -TTGCATAACATTTGGCGGCATCTCTTGACGGAA TCGTCCATTC- $3^{\prime}$. Following primers were used to generate the W407A mutation: forward $5^{\prime}$-CCATAGTTACTTTTCGCCATTGGAATGGAC- ${ }^{\prime}$ ' and reverse $5^{\prime}$-GTCCATTCCAATGGCGAAAAGTAACTATGG-3', as well as forward 5'-CCATAGTTACTTTGCGCCATTGGAATGGAC- ${ }^{\prime}$ ' and reverse $5^{\prime}$-GTCCA TTCCAATGGCGCAAAGTAACTATGG- $3^{\prime}$. After sequencing, the correct plasmids were transformed into E. coli BL21 Rosetta (DE3) cells and expressed and purified as described above.

Kinetic characterization of hTrxR1 variants. The data for the enzyme kinetics were obtained using a U-2100 UV/Vis spectrophotometer (Hitachi). hTrxR1 activity was measured using 5,5'-dithiobis(2-nitrobenzoic acid) (DTNB $)^{22}$ as well as a hTrx1 mutant (C73S $)^{34}$ as substrates. The NADPH-dependent reduction of DTNB/hTrx1 in $100 \mathrm{mM}$ phosphate buffer, $1 \mathrm{mM}$ EDTA, $\mathrm{pH} 7.4$ was determined as the increase/decrease in absorbance at $412 \mathrm{~nm} / 340 \mathrm{~nm}$ at $25^{\circ} \mathrm{C}(\varepsilon 412 \mathrm{~nm}=13.6 \mathrm{~m}$ $\mathrm{M}^{-1} \mathrm{~cm}^{-1} ; \varepsilon 340 \mathrm{~nm}=6.22 \mathrm{mM}^{-1} \mathrm{~cm}^{-1}$ ). For each data point, the initial velocity was determined at least in triplicate over at least 12 different substrate concentrations in 3 independent experiments. Control assays lacking the substrate were routinely included as references.

\section{References}

1. Holmgren, A. et al. Thiol redox control via thioredoxin and glutaredoxin systems. Biochem. Soc. Trans. 33, 1375-1377 (2005).

2. Selenius, M., Rundlof, A. K., Olm, E., Fernandes, A. P. \& Bjornstedt, M. Selenium and the selenoprotein thioredoxin reductase in the prevention, treatment and diagnostics of cancer. Antioxid. Redox Signal. 12, 867-880 (2010).

3. Urig, S. \& Becker, K. On the potential of thioredoxin reductase inhibitors for cancer therapy. Semin. Cancer Biol. 16, 452-465 (2006).

4. Holmgren, A. \& Bjornstedt, M. Thioredoxin and thioredoxin reductase. Methods Enzymol. 252, 199-208 (1995).

5. Lee, S. R. et al. Molecular cloning and characterization of a mitochondrial selenocysteine-containing thioredoxin reductase from rat liver. J. Biol. Chem. 274, 4722-4734 (1999).

6. Turanov, A. A., Su, D. \& Gladyshev, V. N. Characterization of alternative cytosolic forms and cellular targets of mouse mitochondrial thioredoxin reductase. J. Biol. Chem. 281, 22953-22963 (2006).

7. Arner, E. S. Focus on mammalian thioredoxin reductases-important selenoproteins with versatile functions. Biochim. Biophys. Acta 1790, 495-526 (2009). 
8. Lillig, C. H. \& Holmgren, A. Thioredoxin and related molecules-from biology to health and disease. Antioxid. Redox Signal. 9, 25-47 (2007).

9. Turanov, A. A. et al. Mammalian thioredoxin reductase 1: roles in redox homoeostasis and characterization of cellular targets. Biochem. J. 430, 285-293 (2010).

10. Sandalova, T., Zhong, L., Lindqvist, Y., Holmgren, A. \& Schneider, G. Threedimensional structure of a mammalian thioredoxin reductase: implications for mechanism and evolution of a selenocysteine-dependent enzyme. Proc. Natl Acad. Sci. USA 98, 9533-9538 (2001).

11. Karplus, P. A. \& Schulz, G. E. Refined structure of glutathione reductase at 1.54 A resolution. J. Mol. Biol. 195, 701-729 (1987).

12. Berkholz, D. S., Faber, H. R., Savvides, S. N. \& Karplus, P. A. Catcalytic cycle of human glutathione reductase near 1 angstrom resolution. J. Mol. Biol. 382, 371-384 (2008)

13. Biterova, E. I., Turanov, A. A., Gladyshev, V. N. \& Barycki, J. J. Crystal structures of oxidized and reduced mitochondrial thioredoxin reductase provide molecular details of the reaction mechanism. Proc. Natl Acad. Sci. USA 102, 15018-15023 (2005).

14. Lee, S. R. et al. Mammalian thioredoxin reductase: oxidation of the C-terminal cysteine/selenocysteine active site forms a thioselenide, and replacement of selenium with sulfur markedly reduces catalytic activity. Proc. Natl Acad. Sci. USA 97, 2521-2526 (2000).

15. Cheng, Q., Sandalova, T., Lindqvist, Y. \& Arner, E. S. Crystal structure and catalysis of the selenoprotein thioredoxin reductase 1. J. Biol. Chem. 284, 3998-4008 (2009).

16. Fritz-Wolf, K., Urig, S. \& Becker, K. The structure of human thioredoxin reductase 1 provides insights into $\mathrm{C}$-terminal rearrangements during catalysis. J. Mol. Biol. 370, 116-127 (2007).

17. Andersen, J. F. et al. Human thioredoxin homodimers: regulation by $\mathrm{pH}$, role of aspartate 60 , and crystal structure of the aspartate $60 \rightarrow$ asparagine mutant Biochemistry 36, 13979-13988 (1997).

18. Weichsel, A., Brailey, J. L. \& Montfort, W. R. Buried S-nitrosocysteine revealed in crystal structures of human thioredoxin. Biochemistry 46, 1219-1227 (2007).

19. Angelucci, F. et al. Mapping the catalytic cycle of Schistosoma mansoni thioredoxin glutathione reductase by X-ray crystallography. J. Biol. Chem. 285, 32557-32567 (2010).

20. Anestal, K., Prast-Nielsen, S., Cenas, N. \& Arner, E. S. Cell death by SecTRAPs: thioredoxin reductase as a prooxidant killer of cells. PLoS One 3, e1846 (2008).

21. Cheng, Q. et al. The selenium-independent inherent pro-oxidant NADPH oxidase activity of mammalian thioredoxin reductase and its seleniumdependent direct peroxidase activities. J. Biol. Chem. 285, 21708-21723 (2010).

22. Arner, E. S., Zhong, L. \& Holmgren, A. Preparation and assay of mammalian thioredoxin and thioredoxin reductase. Methods Enzymol. 300, 226-239 (1999).

23. Arscott, L. D., Gromer, S., Schirmer, R. H., Becker, K. \& Williams, C. H. Jr The mechanism of thioredoxin reductase from human placenta is similar to the mechanisms of lipoamide dehydrogenase and glutathione reductase and is distinct from the mechanism of thioredoxin reductase from Escherichia coli. Proc. Natl Acad. Sci. USA 94, 3621-3626 (1997).

24. Gromer, S. et al. A hypothesis on the catalytic mechanism of the selenoenzyme thioredoxin reductase. Biochem. J 332, 591-592 (1998).

25. Qiu, W. et al. Dissection of complex protein dynamics in human thioredoxin. Proc. Natl Acad. Sci. USA 104, 5366-5371 (2007).

26. Gasdaska, J. R. et al. Oxidative inactivation of thioredoxin as a cellular growth factor and protection by a Cys73 $\rightarrow$ Ser mutation. Biochem. Pharmacol. 52, 1741-1747 (1996)

27. Ren, X., Bjornstedt, M., Shen, B., Ericson, M. L. \& Holmgren, A. Mutagenesis of structural half-cystine residues in human thioredoxin and effects on the regulation of activity by selenodiglutathione. Biochemistry 32, 9701-9708 (1993).

28. Hashemy, S. I. \& Holmgren, A. Regulation of the catalytic activity and structure of human thioredoxin 1 via oxidation and S-nitrosylation of cysteine residues. J. Biol. Chem. 283, 21890-21898 (2008).
29. Su, D., Berndt, C., Fomenko, D. E., Holmgren, A. \& Gladyshev, V. N. A conserved cis-proline precludes metal binding by the active site thiolates in members of the thioredoxin family of proteins. Biochemistry 46, 6903-6910 (2007).

30. Mitchell, D. A., Morton, S. U., Fernhoff, N. B. \& Marletta, M. A. Thioredoxin is required for S-nitrosation of procaspase-3 and the inhibition of apoptosis in Jurkat cells. Proc. Natl Acad. Sci. USA 104, 11609-11614 (2007).

31. Oblong, J. E., Berggren, M., Gasdaska, P. Y., Hill, S. R. \& Powis, G. Site-directed mutagenesis of Lys36 in human thioredoxin: the highly conserved residue affects reduction rates and growth stimulation but is not essential for the redox protein's biochemical or biological properties. Biochemistry 34, 3319-3324 (1995).

32. Benhar, M., Forrester, M. T., Hess, D. T. \& Stamler, J. S. Regulated protein denitrosylation by cytosolic and mitochondrial thioredoxins. Science 320, 1050-1054 (2008).

33. Urig, S., Lieske, J., Fritz-Wolf, K., Irmler, A. \& Becker, K. Truncated mutants of human thioredoxin reductase 1 do not exhibit glutathione reductase activity. FEBS Lett. 580, 3595-3600 (2006).

34. Irmler, A. et al. in Flavins and Flavoproteins Vol. 14 (eds. Chapman, S., Perham, R. \& Scrutton, N.) 803-815 (Dr. Rudolf Weber, Agency for Scientific Publications, 2002).

35. Kabsch, W. Xds. Acta Crystallogr. D Biol. Crystallogr. 66, 125-132 (2010).

36. Brunger, A. T. et al. Crystallography \& NMR system: A new software suite for macromolecular structure determination. Acta Crystallogr. D Biol. Crystallogr. 54, 905-921 (1998).

37. Adams, P. D. et al. PHENIX: building new software for automated crystallographic structure determination. Acta Crystallogr. D Biol. Crystallogr. 58, 1948-1954 (2002).

38. Jones, T. A., Zou, J. Y., Cowan, S. W. \& Kjeldgaard, M. Improved methods for building protein models in electron density maps and the location of errors in these models. Acta Crystallogr. A 47, 110-119 (1991).

39. Laskowski, R. A., MacArthur, M. W. \& Thornton, J. M. in International Tables of Crystallography Vol. F (eds. Rossmann, M.G. \& Arnold, E.) 722-725 (Kluwer Academic Publishers, 2001).

40. Pettersen, E. F. et al. UCSF Chimera-a visualization system for exploratory research and analysis. J Comput. Chem. 25, 1605-1612 (2004).

\section{Acknowledgements}

We thank Wolfgang Kabsch for helpful discussions as well as Marina Fischer and Dr Rimma Iozef for technical support. Crystallographic data were collected at the Swiss Light Source, beamline X10SA, Paul Scherrer Institute, Villigen, Switzerland SLS. We also thank Ilme Schlichting, Daniel Frey, Anton Meinhard and Bernhard Noll for supporting data collection. The study was supported by the Deutsche Forschungsgemeinschaft (Grant BE 1540/11-1 to K.B.)

\section{Author contributions}

K.F.-W., S.K., S.R. and K.B. contributed to experimental planning and design; K.F.-W., S.K., M.S. performed experimental work and analysed data; S.K., K.F.-W., and K.B. wrote the paper.

\section{Additional information}

Accession codes: Coordinates and structure factors were deposited in Protein Data Bank with the accession codes 3QFA (orthorhombic crystals) and 3QFB (monoclinic crystals).

Supplementary Information accompanies this paper at http://www.nature.com/ naturecommunications

Competing financial interests: The authors declare no competing financial interests.

Reprints and permission information is available online at http://npg.nature.com/ reprintsandpermissions/

How to cite this article: Fritz-Wolf, K. et al. Crystal structure of the human thioredoxin reductase-thioredoxin complex. Nat. Commun. 2:383 doi: 10.1038/ncomms1382 (2011). 